\title{
Modelling of OH/IR Dust Envelopes
}

\section{S. LORENZ MARTINS and F. X. DE ARAÚJO}

\author{
Observatorio Nacional-DAGE, Rio de Janeiro, Brazil
}

One of the least understood phases of stellar evolution occurs at the tip of the AGB and immediatly following the AGB. The stellar population at the tip of the AGB consists mainly of OH/IR stars and carbon stars. These classes are distinguished by the oxygen-rich envelope of $\mathrm{OH} / \mathrm{IR}$ stars and the carbon-rich envelope of carbon stars. It is thought that both kinds of stars evolve from Mira variables and both can be progenitors of planetary nebulae. $\mathrm{OH} / \mathrm{IR}$ objects can be classified in two groups. Type I OH/IR stars have lower mass-loss rates and they are frequently identified optically as Mira variables.

We have performed calculations of radiative transfer in circumstellar dust shells and the modelling of $19 \mathrm{OH} / \mathrm{IR}$ objects (types I and II). The Monte Carlo method was employed for representing the propagation of radiative energy, photon by photon, and solving the radiative transfer. We have modified previous versions of the numerical code in order to allow choosing the distribution of matter in the envelope, say the density law, while maintaining spherical symmetry. Then, we have modelled our sample considering $\rho \propto r^{-2.0}$, $\rho \propto r^{-2.5}$, and $\rho \propto r^{-1.3}$. Spherical grains with different radii in the range $900-8000 \AA$ have been considered. The absorption and scattering efficiencies, as well as the albedo of the grains, were calculated using the Mie theory and the optical constants of David \& Pégourié (1995, A\&A, 293, 833). These constants are essential for building the models. The temperature of the central star and some characteristics of the circumstellar envelopes were determined by fitting the observed flux curves. Preliminary results show that type I OH/IR stars have thinner dust envelopes than type II ones. The temperatures also differ according to the types: type I OH/IR stars have higher temperatures than type II OH/IR stars. These results could indicate that type I OH/IR stars are less evolved than type II OH/IR stars.

This work was supported by CNPq-Brazil. 